INRA Prod. Anim., 1996, 9 (4), 243-254

\section{T. GIDENNE}

INRA Station de Recherches Cunicoles, BP 27, 31326 Castanet-Tolosan Cedex
Conséquences digestives de l'ingestion de fibres et d'amidon chez le lapin en croissance : vers une meilleure définition des besoins

Accroître la teneur en énergie digestible des régimes pour lapins en croissance passe souvent par l'augmentation de leur teneur en amidon et la diminution de leur teneur en fibres. Mais un apport minimum de fibres est indispensable pour assurer un bon fonctionnement digestif. Même avec cet apport minimum, des troubles digestifs peuvent survenir si l'apport d'amidon est trop élevé. L'équilibre entre bonnes performances de croissance et bon état sanitaire nécessite donc de définir non seulement l'apport minimum de fibres, mais aussi l'apport maximum d'amidon.

\section{Résumé}

Le lapin peut utiliser efficacement des aliments peu fibreux, à teneur élevée en énergie digestible. Mais la réduction de la teneur en fibres des régimes, associée le plus souvent à une élévation de la teneur en amidon, conduit à une augmentation de la fréquence des troubles digestifs (souvent mortels). Il faut donc maîtriser les apports alimentaires de fibres et d'amidon, aux plans quantitatif et qualitatif, pour permettre une croissance et une sécurité alimentaire optimales. Un apport de lignocellulose (ADF), faiblement dégradable, entraîne une réduction de la digestibilité du régime et stimule le transit digestif, mais modifie peu les fermentations caecales. Un apport de fibres relativement digestible (hémicelluloses + pectines), affecte modérément la digestion de l'aliment, n'entraîne pas de ralentissement du transit digestif et favorise l'activité microbienne caecale. Au plan de la santé de l'animal, un apport d'ADF paraît efficace pour réduire la fréquence des troubles digestifs et la mortalité pendant la période d'engraissement.

L'activité microbienne caecale peut aussi être influencée par la quantité d'amidon entrant dans le caecum, elle-même dépendante de la nature de l'amidon et de l'âge de l'animal. L'augmentation de la teneur en amidon d'un régime peut favoriser l'apparition de troubles digestifs, même lors d'un apport d'ADF conforme aux recommandations actuelles.

Ainsi, recommander un apport minimum d'ADF n'est pas suffisant pour assurer la sécurité d'un aliment, il faut y associer une norme d'apport maximum d'amidon. Le remplacement d'amidon par des constituants pariétaux à digestibilité élevée, dans un régime contenant un apport minimum de lignocellulose, pourrait être une solution à l'antagonisme entre efficacité et sécurité alimentaire chez le lapin en croissance.
En tant qu'herbivore, le lapin possède une physiologie digestive adaptée à l'ingestion de quantités élevées de fibres alimentaires, qu'il digère avec une efficacité modeste. Cependant, le lapin est aussi un monogastrique et il utilise très efficacement des aliments peu fibreux, "riches » en céréales, et dont la teneur en énergie digestible est plus élevée. Depuis les années 70 , la rationalisation de l'alimentation a conduit à une élévation de la teneur en énergie digestible des aliments afin d'accroître les performances de croissance. Mais cette hausse de la concentration énergétique est le plus souvent obtenue par le biais d'une élévation de la teneur en amidon aux dépens de la teneur en fibres. Ceci peut conduire à des dysfonctionnements digestifs graves, en particulier chez le lapin en croissance : altérations des fermentations caecales, modification de la motricité digestive, diarrhées. Un apport alimentaire minimum de parois végétales est donc indispensable pour assurer un fonctionnement digestif normal (Laplace 1978), et éviter ainsi l'apparition d'entérites souvent mortelles (Peeters et Charlier 1984). Ce besoin en fibres limite donc les possibilités d'accroissement de la 
teneur en énergie des régimes et, par voie de conséquence, leur efficacité alimentaire. Cet antagonisme entre performances de croissance et bon état sanitaire constitue un problème majeur en nutrition cunicole. Ainsi, les résultats de la gestion technico-économique indiquent une mortalité en engraissement d'environ $12 \%$ (Kœhl 1995), dont une part importante est attribuable à des entérites non spécifiques.

Beaucoup de travaux ont été réalisés antérieurement, afin d'analyser les effets de la nature et/ou de la quantité de fibres alimentaires sur la digestion chez le lapin. Mais les apports de fibres varient généralement à l'inverse des apports d'amidon (fibres et amidon constituant les $2 / 3$ d'un régime), et peu d'études ont été effectuées sur les conséquences digestives de l'ingestion d'amidon (nature et/ou quantité) et sur les interactions avec les apports de fibres. Nous nous proposons ici de faire le point des connaissances sur les conséquences de l'ingestion de fibres et d'amidon sur la digestion du lapin, en traitant successivement des effets spécifiques des fibres, puis des interactions avec les apports d'amidon. Mais, auparavant, il nous paraît utile de présenter un bref rappel des caractéristiques biochimiques des fibres et des fractions fibreuses obtenues par les méthodes d'analyses applicables à l'alimentation animale.

\section{1 / Définitions et rappels}

\section{1 / Caractéristiques biochimiques des fibres alimentaires et conséquences sur leur analyse}

$\mathrm{Au}$ plan de la structure biochimique, il existe de nombreuses classes de fibres, dont la plupart sont des constituants de la paroi des cellules végétales. Il est cependant possible de distinguer 5 classes majeures (figure 1) : lignines, cellulose, hémicelluloses insolubles dans l'eau, substances pectiques insolubles dans l'eau, et une classe de divers polysaccharides non amylacés hydrosolubles.

On peut définir les fibres alimentaires, comme la somme des polysaccharides non amylacés et des lignines. Ces polymères végétaux ne sont pas hydrolysables par les enzymes endogènes de l'animal, en raison de la nature des liaisons chimiques entre les monomères. Par contre, la flore bactérienne, présente essentiellement dans le caecum et le côlon proximal du lapin, possède les enzymes permettant l'hydrolyse des chaînes polysaccharidiques en oses simples, dont la fermentation conduit à une production d'acides gras volatils (absorbés par la paroi caecale) et de $\operatorname{gaz}\left(\mathrm{CO}_{2}, \mathrm{CH}_{4}, \mathrm{H}_{2}\right)$.

La structure détaillée de ces différentes classes de fibres a déjà fait l'objet de synthèses (Aspinall 1980, Selvandran 1987), nous en ferons ici un bref rappel. Les polysaccharides solubles dans l'eau comprennent plusieurs classes de molécules de degrés de polymérisation d'environ 15 à plus de 2000 ( $\beta$-glucanes). Les substances pectiques recouvrent plusieurs classes de polymères, incluant les pectines - constituées d'un squelette rhamnogalacturonique et de chaînes latérales d'arabinose et de galactose, mais aussi des polysaccharides neutres (arabinanes, galactanes, arabinogalactanes), souvent associés aux pectines. Les hémicelluloses regroupent plusieurs types de polysaccharides, tels que les xylanes, les mannanes (chaînes linéaires, liaisons $\beta 1-4$ ), les arabino-galactanes (chaînes ramifiées, liaisons $\beta 1-3$ et $\beta 1-6)$. Par contre, la cellulose est un homopolymère linéaire de glucose (liaisons $\beta 1-4$ ), qui s'agrège en microfibrilles formant ainsi le squelette de la paroi végétale et donc de la plante. Les lignines sont des constituants pariétaux non glucidiques (Monties 1980). Ce sont des polymères phénoliques organisés en réseaux très complexes. Plus globalement, la paroi végétale est donc constituée de fibrilles de cellulose (squelette) intégrées dans une matrice composée de lignines, hémicelluloses, pectines et protéines.

Compte tenu de la diversité des molécules composant les fibres alimentaires, il n'y a actuellement aucune méthode de dosage permettant un fractionnement correct des diffé-

Figure 1. Les principales classes de fibres alimentaires.

\begin{tabular}{|c|c|c|c|}
\hline \multirow{3}{*}{$\begin{array}{l}\text { Fibres } \\
\text { alimentaires totales }\end{array}$} & & Lignines (1) & \multirow{3}{*}{ Fibres insolubles dans l'eau } \\
\hline & & Cellulose (1) & \\
\hline & non amylacés (PNA) & Hémicelluloses (1) & \\
\hline \multirow{2}{*}{ (Total Dietary Fibre, TDF) } & $\begin{array}{l}\text { (Non Starch Poly- } \\
\text { saccharides, NSP) }\end{array}$ & Substances pectiques (1) & \\
\hline & & $\begin{array}{l}\text { PNA hydrosolubles: } \\
\text { pectines hydrosolubles (luzerne), } \\
\beta \text {-glucanes (orge), } \\
\text { arabinoxylanes (blé) }\end{array}$ & $\begin{array}{l}\text { Fibres solubles dans l'eau } \\
\text { (Water soluble NSP) }\end{array}$ \\
\hline
\end{tabular}


Figure 2. Méthodes gravimétriques de dosage des fibres et nature du résidu d'analyse.

(1) TDF = total dietary fibre (Lee et al 1992) ; (2) WICW = water insoluble cell-wall (Carré et Brillouet 1989) ;

(3) $N D F=$ neutral detergent fibre, $A D F=$ acid detergent fibre, $A D L=$ acid detergent lignin

(Van Soest et al 1991); (4) selon Weende.

Résidu d'analyse

Polymères
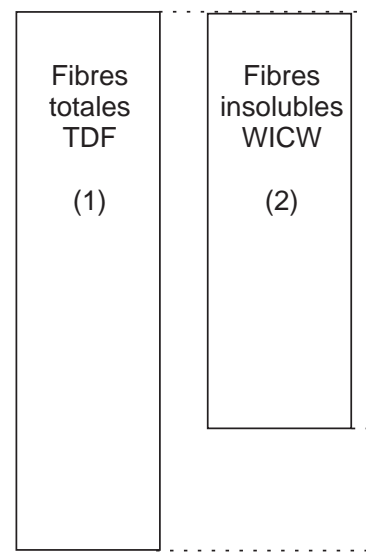
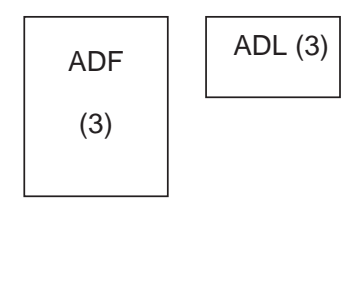
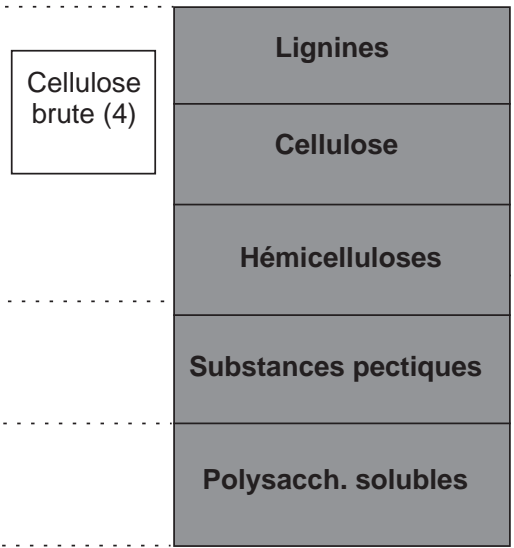

rentes classes de fibres. Il existe cependant des méthodes, plus ou moins précises, pour isoler certaines classes de fibres dans les aliments (figure 2), dont les procédures ont fait l'objet d'articles de synthèse (Asp et Johansson 1984, Carré 1991, Van-Sœst et al 1991, Giger-Reverdin 1995). Les résultats d'analyse des fibres dans les aliments et matières premières destinés aux animaux correspondent donc rarement à des classes de fibres précises. Rappelons le cas de la méthode Weende, utilisée en routine en alimentation cunicole, pour définir les recommandations en fibres. Cette technique permet d'isoler un résidu fibreux, la «cellulose brute", qui correspond selon les matières premières à une fraction plus ou moins importante de la cellulose et des lignines, et qui n'inclut pas les hémicelluloses et les substances pectiques. En fonction de la technique de dosage, l'estimation de la part des fibres dans un aliment pour lapins pourra évidemment varier fortement (tableau 1).

\section{2 / Facteurs de variation de la digestion des fibres par le lapin}

Remarquons au préalable que la mesure de la teneur en fibres des fèces et des digesta, lors d'études de digestion, est peu précise car les méthodes de dosage ont été mises au point

Tableau 1. Proportions courantes de fibres dans un aliment complet destiné au lapin en croissance.

\begin{tabular}{|l|c|}
\hline \multicolumn{1}{|c|}{ Résidu d'analyse } & Proportion (\%) \\
\hline Cellulose brute (CB) & 14 à 18 \\
Acid Detergent Fibre (ADF) & 15 à 20 \\
Neutral Detergent Fibre (NDF) & 25 à 40 \\
Parois végétales insolubles (WICW) & 27 à 45 \\
Fibres totales (TDF) & 30 à 48 \\
\hline Autres constituants alimentaires \\
\hline Amidon & 10 à 20 \\
Matières azotées totales (N x 6,25) & 13 à 18 \\
\hline
\end{tabular}

pour les aliments. Ainsi, les valeurs de digestibilité des constituants pariétaux relevées dans la littérature sont souvent très variables, et il convient d'être prudent quant à leur validité.

Le facteur de variation principal de la digestion des fibres est tout d'abord leur nature. Dans la plupart des études, la technique de Van Soest a été employée afin d'estimer la digestibilité des différentes fractions de fibres. En règle générale, les hémicelluloses (NDF-ADF) présentent une dégradation supérieure à celle de la cellulose (ADF-ADL), mais nettement inférieure à celle des substances pectiques (estimées en dosant les acides uroniques) (tableau 2). Soulignons que cette hiérarchie correspond à celle des activités fibrolytiques bactériennes du caecum (Marounek et al 1995) : cellulase < xylanase < pectinase. Les lignines, théoriquement indigestibles, présentent chez le lapin des coefficients de digestibilité très variables, du fait de l'imprécision des méthodes de dosage et de la faible teneur en lignines des aliments (environ $5 \%$ ), et aussi du fait de leur origine très variée (cf 2.2).

Les variations de digestibilité des fibres liées à l'animal (âge, race) sont de moins grande ampleur que celles liées à la nature des fibres. L'adaptation morpho-fonctionnelle du système digestif au cours de la croissance (réservoirs digestifs plus volumineux, installation de la flore, etc) pourrait être un facteur important des variations de digestion des fibres. Cependant, chez le lapin, cette question fait l'objet de controverses. Ainsi, selon les études, la digestibilité des fibres augmente ou diminue entre le sevrage et l'âge à l'abattage (Gidenne et Perez 1993a).

\section{2 / Effets spécifiques des fibres sur la digestion chez le lapin}

Nous avons vu précédemment qu'il existait différentes classes de fibres, dont la nature

\section{La digestion des fibres dépend surtout de leur nature : les hémicelluloses sont mieux digérées que la cellulose mais nettement moins que les substances pectiques.}


biochimique pouvait être très variée. Il convient donc de décrire les effets digestifs des fibres liés soit à une variation de la quantité de fibres ingérées, sans variation de leur nature, soit à la nature des fibres.

Pour ces deux aspects, nous examinerons successivement les effets des fibres sur les trois fonctions digestives principalement concernées : la digestion des nutriments, l'activité microbienne caecale et le transit digestif. Dans la mesure des résultats disponibles, nous évoquerons également les conséquences des fibres sur l'état sanitaire des animaux.

\section{1 / Incidence de la quantité de fibres ingérées}

Peu d'études ont été consacrées strictement aux effets liés à la quantité de fibres ingérée, sans variations de la proportion des différents constituants pariétaux (cellulose, lignines, etc). Il y a souvent eu une confusion entre quantité et nature des fibres apportées par l'aliment. L'emploi de la méthode de Weende pour mesurer les apports de fibres est sans doute une des causes majeures de cette confusion, puisque cette méthode ne permet d'analyser qu'une fraction des fibres présentes dans l'aliment. Au-delà du problème de l'analyse chimique des fibres, il conviendrait de comparer des aliments qui se différencient seulement par le taux d'incorporation des mêmes sources de fibres, pour étudier les effets stricts de la quantité de fibres ingérée.

Rappelons qu'en conditions courantes d'élevage le lapin est alimenté à volonté, et qu'il ajuste globalement sa consommation en fonction de la teneur en énergie digestible (ED) du régime. Une réduction de la teneur en fibres (équivalent à une hausse de l'ED) entraîne ainsi une réduction de l'ingestion volontaire, et il faut donc évaluer quelle en est la répercussion sur l'ingestion des différents constituants alimentaires pour s'assurer de l'origine des effets digestifs observés. Ainsi, lorsque la teneur en NDF baisse de 39 à $22 \%$, l'ingestion de fibres baisse de $18 \mathrm{~g} / \mathrm{j}$ (soit - 58\%) sous l'effet conjugué de la réduction de la teneur en fibres et de la baisse du niveau d'ingestion d'aliment, tandis que la quantité d'amidon ingérée n'augmente que de $6 \mathrm{~g} / \mathrm{j}$. Dans ces conditions, les effets digestifs proviennent bien pour l'essentiel de variations de la quantité de fibres ingérée, et non de celle d'amidon.

Tableau 2. Valeurs de digestibilité des fibres dans l'ensemble du tube digestif du lapin (données bibliographiques).

\begin{tabular}{|l|c|c|}
\hline \multirow{2}{*}{} & \multicolumn{2}{|c|}{ Digestibilité fécale $(\%)$} \\
\cline { 2 - 3 } & moyenne & mini-maxi \\
\hline Lignines (ADL) & 10 à 15 & -13 à +50 \\
Cellulose (ADF-ADL) & 15 à 18 & 4 à 37 \\
Hémicelluloses (NDF-ADF) & 25 à 35 & 11 à 60 \\
Pectines (Ac. uroniques sol. + insol.) & 70 à 76 & nc \\
\hline
\end{tabular}

nc $\square$ : non calculé, en raison d'un trop faible nombre de données.
Figure 3. Digestibilité de la matière organique et des matières azotées totales (MAT, $N \times 6,25)$ en fonction de la teneur en lignocellulose $(A D F) d u$ régime, chez le lapin en croissance (d'après Gidenne et al 1991, Bellier 1994).

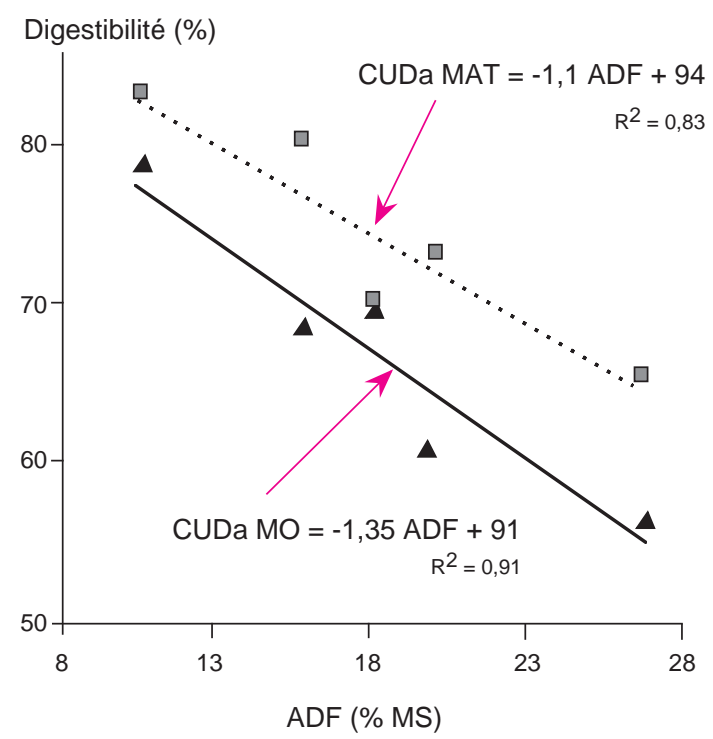

Lorsque la teneur en fibres d'un aliment diminue, en substituant de l'amidon, on observe généralement une augmentation de la digestibilité fécale de la matière organique (MO). A titre d'exemple, les résultats de deux expérimentations (Gidenne et al 1991, Bellier 1994) indiquent que la digestibilité de la MO augmente de 1,35 points lorsque la teneur en $\mathrm{ADF}$ diminue de 1 point (figure 3 ). Le coefficient de - 1,35 n'indique évidemment pas que la fraction ADF possède une digestibilité « négative ", mais que l'apport d'ADF a un effet négatif sur la digestion. Il y a ici un phénomène de compensation entre la digestibilité positive de $\mathrm{ADF}$ (en moyenne 15 à $20 \%$ ), et un abaissement global de la digestibilité du fait d'une plus grande proportion de constituants cellulaires provenant de sources de fibres (effet barrière de la paroi végétale, causé en particulier par les lignines). On peut calculer également que la diminution du CUD de la MO n'est que de 0,98 point par point de $\mathrm{NDF}$ ajouté (CUD MO $=-0,98 \mathrm{NDF}+96,2$; $\left.\mathrm{R}^{2}=0,78\right)$. Le coefficient égal à 0,98 indique que globalement l'apport de NDF a un effet neutre (rôle de diluant) sur la digestion de la ration (mais n'indique pas une digestibilité nulle de NDF), ainsi que cela a été décrit antérieurement pour le porc (Perez 1990). En effet, par rapport à ADF, le résidu NDF contient en sus les hémicelluloses, mieux digérées par le lapin que la cellulose (tableau 2 ), d'où une moindre baisse de digestibilité de la ration. Des résultats similaires ont été obtenus par De Blas et al (1986), mais cet effet des fibres sur la digestibilité dépend aussi largement de la proportion respective des différentes fractions pariétales (lignines, cellulose, etc) dont la digestibilité est différente (cf 2.2). Par exemple, si l'apport de 
fibres est principalement constitué de lignocellulose (Partridge et al 1989), la baisse de digestibilité de l'aliment par point d'ADF sera élevée (- 1,7 points par point d'ADF). C'est pourquoi les équations de prédiction de la digestibilité d'un régime basées uniquement sur un seul critère d'analyse chimique des fibres alimentaires (ADF ou cellulose brute) sont imprécises (Maertens et al 1988, Ortiz et De Blas 1989). Par ailleurs, la digestibilité des matières azotées totales (MAT) baisse d'environ un point par point d'ADF (figure 3).

Lors d'une baisse de la teneur en fibres de l'aliment, la quantité quotidienne de fibres dégradée (g NDF digéré/j) décroît généralement, en raison d'une forte baisse de l'ingestion de fibres. Ceci correspond à une moins forte activité microbienne caecale, mais qui ne se traduit pas toujours par une baisse de la concentration des acides gras volatils (AGV) dans le caecum (Gidenne et al 1991, Bellier et Gidenne 1996). L'intensité des fermentations caecales ne serait donc pas limitée par la teneur en fibres, mais dépendrait d'avantage de leur nature (cf 2.2). Par contre, la baisse de la teneur en fibres du régime agit plus clairement sur l'orientation des fermentations, avec pour effet majeur d'élever la proportion molaire de butyrate. L'activité fermentaire caecale peut dépendre également du transit digestif. En effet, des lapins nourris avec un régime peu fibreux, mais adaptés préalablement à un régime riche en fibres, présentent un temps de séjour moyen dans l'ensemble du tube digestif (TSMt) plus bref ( $-20 \%)$, et une concentration caecale d'AGV plus élevée $(+50$ $\%$ ), que des lapins constamment nourris avec le régime peu fibreux (Gidenne et al 1991).

$\mathrm{Au}$ plan du transit digestif, la réduction de l'ingestion de fibres a pour effet d'allonger le TSMt des aliments (Lebas et Laplace 1977, Fraga et al 1984, Gidenne et al 1991). Ainsi, pour une baisse de l'ingestion de NDF de 57 à $22 \mathrm{~g}$ NDF/j, le TSMt passe de 17 à $33 \mathrm{~h}$ chez le lapin en croissance (Bellier et Gidenne 1996). Ce ralentissement du transit peut être en partie expliqué par une baisse du niveau d'ingestion de l'aliment. Cependant, chez des lapins adultes dont l'ingestion volontaire $(115 \mathrm{~g} / \mathrm{j})$ a peu varié malgré une diminution de la teneur en fibres (40 à $21 \%$ NDF), Gidenne (1994) a observé une augmentation du TSMt de 16 à $28 \mathrm{~h}$ (figure 4). Il y a donc bien un effet spécifique de la quantité de fibres ingérée sur le transit, indépendamment des variations de niveau d'ingestion de l'aliment. De plus, l'effet des fibres est différent en fonction du segment digestif concerné. Ainsi, la baisse de l'apport de fibres entraîne un allongement important $\mathrm{du}$ transit caecal (figure 4) et un transit stomacal plus court. Cependant le transit oroiléal n'est pas modifié, car l'accélération de la vidange gastrique est compensée par un transit intestinal plus lent. Il y a donc, chez le lapin, une régulation du transit oro-iléal en fonction de l'ingestion de fibres, mais l'effet majeur des fibres porte surtout sur le transit cæcal.
Figure 4. Incidence d'une baisse de la quantité de fibres ingérée sur le transit dans les différents compartiments digestifs du lapin (d'après Gidenne 1994).

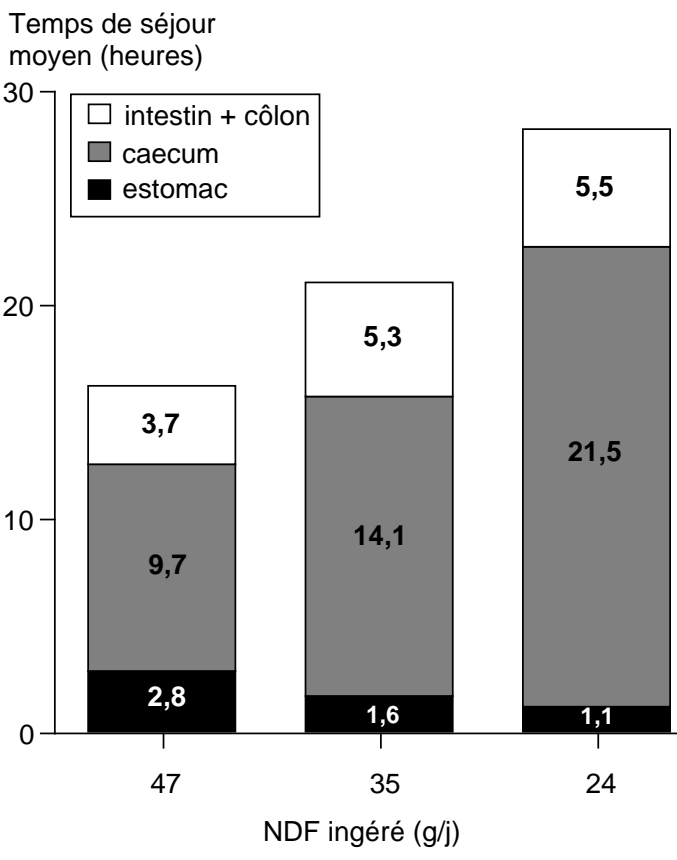

Au plan de la santé du lapin, une réduction de l'apport de fibres provoque une fréquence plus élevée de troubles digestifs chez le lapin en croissance (Heckmann et Mehner 1970, Franck et Coulmin 1978, Blas et al 1994). Cependant, très peu d'études ont tenté d'analyser l'effet strict de la quantité de fibres ingérée (sans variation de leur nature) sur la mortalité. La nécessité d'utiliser un nombre élevé de lapins par traitement, pour mesurer la fréquence des troubles digestifs, est sans doute une des raisons du faible nombre de travaux réalisés. Ainsi, lors d'une baisse de la teneur en $\mathrm{ADF}$ de 14,5 à 9,3\%, Gidenne (1995) observe, pour des groupes de 40 lapins par régime, un accroissement de la morbidité de 19 à $28 \%$, mais qui demeure non significatif $(\mathrm{P}=0,14)$

Figure 5. Incidences d'une déficience en fibres alimentaires sur quelques paramètres de la digestion chez le lapin.

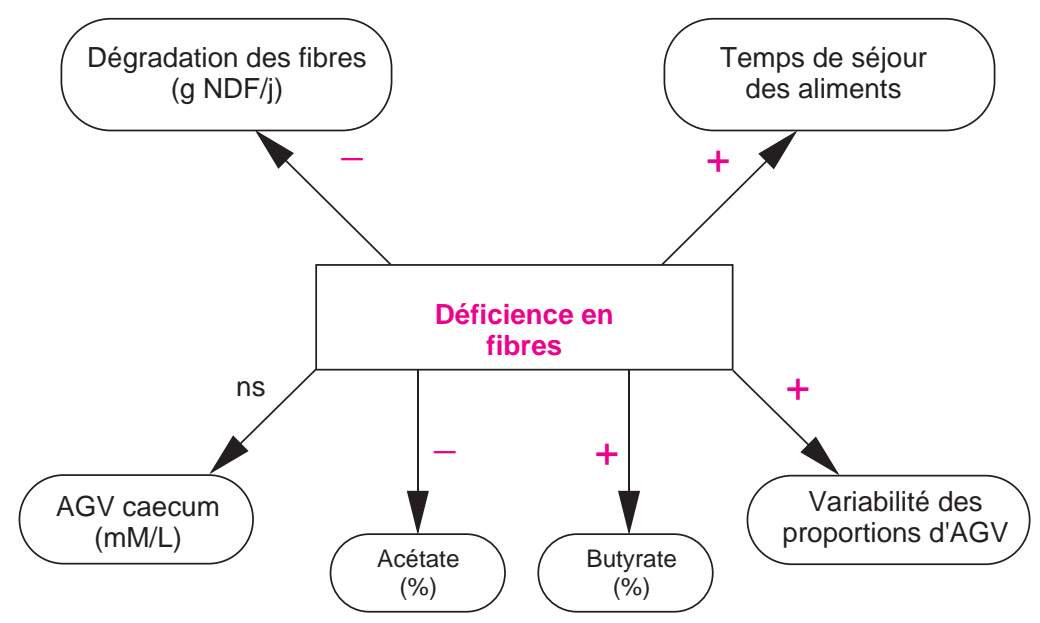

A même quantité d'aliment ingérée, diminuer sa teneur en fibres augmente le temps de séjour dans le tube digestif. 
Pour résumer (figure 5), un apport alimentaire déficient en fibres conduit à un ralentissement du transit digestif et à une baisse de la quantité de fibres dégradée. En outre, cela modifie l'orientation des fermentations caecales, dont la variabilité inter-animal s'accroît (Bellier et Gidenne 1996). Ce dernier point suggère une plus forte sensibilité de l'activité microbienne aux conditions de milieu, en particulier vis-à-vis d'agents pathogènes éventuels.

\section{2 / Incidence de la nature des fibres ingérées}

Chaque matière première végétale possède un profil spécifique en fibres, qui correspond aux proportions des différents constituants de la paroi des cellules végétales (figure 6). De plus, ce profil évolue avec la croissance de la plante : citons pour exemple l'accroissement de la teneur en lignines de la paroi végétale lorsque la plante vieillit. En fonction du profil en fibres des matières premières incorporées dans un aliment, nous observerons donc des effets digestifs spécifiques chez l'animal. Nous distinguerons deux parties dans ce paragraphe : d'une part les études ayant pour objet la valeur nutritive de matière premières sources de fibres, et d'autre part l'étude des effets digestifs liés plus strictement aux variations d'un constituant pariétal (ex : les lignines).

\section{a / Effets liés aux sources de fibres}

De nombreux travaux ont été effectués pour étudier in vivo la valeur nutritive des sources de fibres, car l'analyse chimique des fibres ( souvent réduite à la cellulose brute) est insuffisante pour apprécier et prévoir les effets digestifs chez l'animal. De plus, l'alimentation cunicole utilise de nombreux sousproduits agro-alimentaires fibreux (pulpes, coques de graines, etc), dont il faut estimer la valeur nutritive (Motta Ferreira 1990, Perez de Ayala et al 1991, Merino et Carabaño 1992). Ainsi, Candau et al (1978a) soulignent la meilleure digestibilité des fibres de pulpes de betteraves (CUDa ADF $=53 \%$ ) comparées à celles de la luzerne déshydratée (CUDa $\mathrm{ADF}=20 \%$ ) pour des régimes à même taux de cellulose brute. Mais la lignification des parois végétales est réduite dans le cas des pulpes, ce qui permet une meilleure dégradation de la cellulose et des hémicelluloses. A l'inverse, les fibres de paille de blé, plus lignifiées, ne sont que faiblement dégradées (CUD $\mathrm{ADF}=14 \%$, De Blas et al 1989).

La valeur nutritive des sources de fibres peut aussi dépendre de leur taux d'incorporation dans l'aliment. Par exemple, le mélange de pulpes de betterave à du foin de luzerne accroît la digestibilité de la luzerne (Gidenne 1987), ou encore la valeur de la pulpe de betterave est plus faible lorsque le rapport fibre/amidon du régime augmente (De Blas et Carabaño 1996). L'évaluation de la valeur nutritive des sources de fibres doit donc tenir compte de ces phénomènes d'interactions digestives.
Figure 6. Exemple de composition en constituants pariétaux (\% des fibres insolubles) quelques sources de fibres utilisées en alimentation cunicole.

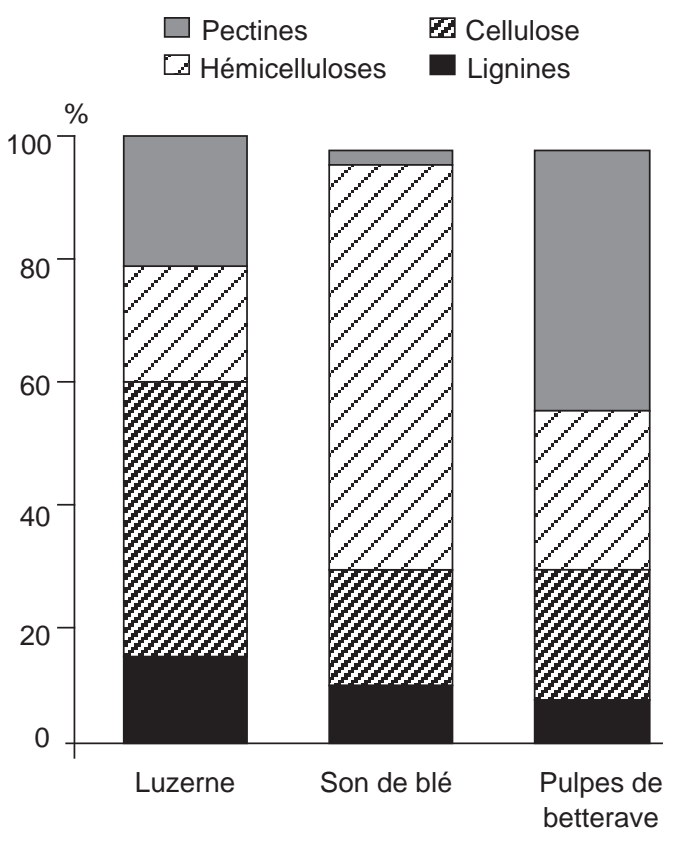

Le transit digestif est influencé par la source de fibres. Ainsi, une source de fibres lignifiées (marc de raisin) entraîne une forte réduction du TSMt (Fraga et al 1991). Certains auteurs ont avancé qu'une source de fibres plus digestibles telle que la pulpe de betterave pourrait à l'inverse ralentir le transit, alors que les résultats expérimentaux n'indiquent pas d'allongement du TSMt mais plutôt un effet neutre ou de réduction du TSMt (Gidenne et al 1987, Fraga et al 1991, Bellier 1994). Il faut aussi signaler que la forme physique de la source de fibres, en particulier la granulométrie, peut influencer le transit. Ainsi, le broyage d'une même matière première conduit à un allongement du TSMt, mais sans modifier notablement les fermentations caecales (Laplace et Lebas 1977, Gidenne et al 1990).

L'activité fermentaire caecale est également influencée par la source de fibres. L'incorporation dans un aliment de matières premières riches en pectines (pulpes de betterave) entraîne une élévation de la concentration des AGV (Candau et al 1978b, Jehl et Gidenne 1996). De plus, l'origine botanique des fibres semble aussi influencer l'activité fermentaire caecale, même lorsque le taux de fibre et les proportions des différentes fractions pariétales (méthode de Van Sœst) sont similaires (Bellier et Gidenne 1995).

L'incidence de l'incorporation de sources de fibres sur la mortalité du lapin en croissance a été souvent étudiée avec un nombre restreint d'animaux ( $\mathrm{n}<40 /$ régime). On peut cependant citer les travaux de Franck et Seroux (1980) et de Motta Ferreira (1990), qui ne montrent aucun effet sur la mortalité en engraissement de l'incorporation à taux élevés (jusqu'à $60 \%$ ) de pulpes de betterave (régimes iso-ADF). En revanche, l'incorpora- 
tion de plus de $45 \%$ de puples de citron dans aliment entraîne une hausse significative de la mortalité (Martinez Pascual et Fernandez Carmona 1981).

\section{b / Effets liés à la nature des constituants pariétaux}

Nous traiterons ici des effets digestifs observés lors de variations de la teneur d'un constituant pariétal sans variations importantes des autres classes de fibres: par exemple, accroissement de l'apport de lignines, sans variation des apports de cellulose ou d'hémicelluloses. Contrairement aux résultats précédents sur les effets des sources de fibres, les variations d'un type de constituant pariétal sont obtenues en combinant un nombre assez élevé de matières premières. Par ailleurs, rappelons qu'en alimentation animale, les différentes fractions pariétales sont analysées selon la technique de Van Sœst. La détermination des taux de cellulose (ADF-ADL) ou des hémicelluloses (NDF-ADF) est donc obtenue par différence entre deux résidus fibreux, et peut être l'objet d'imprécisions non négligeables. D'autre part, le système Van Soest ne tient pas compte des substances pectiques ni des polysaccharides non amylacés hydrosolubles.

La réduction de 21 à $15 \%$ de la teneur en lignocellulose (ADF), conduit à une meilleure digestibilité de l'aliment, mais ceci est associé à une hausse de la mortalité en engraissement de 7,5 à 14,4 \% (Maître et al 1990). Afin de déterminer le rôle respectif des lignines et de la cellulose dans la fraction $\mathrm{ADF}$, d'autres travaux ont été conduits, en particulier sur les lignines. Ces dernières ne sont pas dégradées par les bactéries, elles sont donc théoriquement indigestibles et constituent un obstacle à la dégradation des autres constituants de la paroi végétale. Pourtant, chez le lapin, les lignines de certains sous - produits agroalimentaires (peaux de tomate, marc de raisin, etc) pourraient avoir un effet positif sur la digestibilité des fibres (Falcao e Cunha 1988), et sur la concentration des AGV dans le caecum (Gidenne 1986). Mais, dans ce cas, la fraction lignine analysée inclut en général d'autres composés tels que des tanins ou des cutines, il est donc difficile d'attribuer strictement aux lignines les effets digestifs observés. Lorsque les lignines sont fournies par des matières premières plus classiques (luzerne, paille...), on observe un effet négatif des lignines sur la digestion, associé à un transit digestif plus court (Gidenne et Perez 1994). On observe aussi une réduction linéaire du taux de mortalité avec l'accroissement de l'apport de lignines (Perez et al 1994).

A notre connaissance, seule Falcao e Cunha (1988) a étudié les effets digestifs en relation avec les apports de cellulose (ADF-ADL). La réduction de la teneur en cellulose de 11,5 à $7,5 \%$ améliore la digestion du régime et des fibres (NDF), mais n'intervient ni sur la caecotrophie ni sur le développement du caecum, et ne modifie pas le $\mathrm{pH}$ caecal. Des travaux complémentaires devront donc être réalisés pour préciser le rôle de la cellulose sur le transit digestif et la mortalité. Enfin, une seule étude a concerné les effets d'une réduction des apports d'hémicelluloses, sans variation importante des autres fractions pariétales (Lebas et al 1989) : une légère hausse de la digestibilité du régime est observée ; la mortalité en engraissement n'est pas affectée par la teneur en hémicelluloses, mais pourrait être influencée par l'origine des hémicelluloses.

Plus globalement, un apport de fibres de type lignocellulosique (ADF), peu digestibles, entraîne une réduction de la digestibilité de l'aliment (et donc une baisse des performances de croissance), associé à un transit digestif plus rapide, mais sans modification importante du niveau des fermentations caecales. Un apport de fibres de type hémicellulosique ou pectique, relativement digestibles, réduit peu la digestion de l'aliment, semble efficace pour réguler le transit digestif, et favorise l'activité fermentaire cæcale. Au plan de la santé de l'animal, un apport de lignocellulose apparaît plus efficace que les hémicelluloses pour réduire la fréquence des troubles digestifs et la mortalité en engraissement.

\section{3 / Interactions entre apports de fibres et d'amidon}

\section{1 / Digestion de l'amidon et conséquences sur la digestion des fibres}

Il est important de connaître les facteurs qui interviennent sur le site de dégradation de l'amidon, et en particulier la quantité et la nature de l'amidon ingéré, en rapport avec les capacités d'hydrolyse intestinale de l'amidon par l'animal. En effet, une surcharge alimentaire en amidon peut accroître le flux d'amidon entrant dans le caecum, modifier l'activité microbienne, et pourrait être à l'origine de troubles digestifs chez le lapin en croissance (Cheeke et Patton 1980). L'emploi d'amidon peu digeste dans l'intestin grêle peut aussi être un facteur d'accroissement du flux iléal d'amidon. Par exemple, l'incorporation de plus de $20 \%$ de farine de pomme de terre entraîne une hydratation des fèces, susceptible de favoriser l'apparition de troubles digestifs (Heckmann 1972).

L'étude des conséquences digestives de l'ingestion de l'amidon chez le lapin a fait l'objet de travaux relativement récents et peu nombreux, comparativement à ceux menés sur les effets des fibres. Chez le lapin adulte ou en fin de croissance, la digestibilité fécale de l'amidon est quasi-totale (95 à $100 \%$ ). On observe généralement une légère baisse de la digestibilité fécale de l'amidon lorsque sa concentration dans l'aliment augmente (Parigi-Bini et al 1990), que ce soit de l'amidon d'orge ou de maïs (Blas et al 1990). Mais la digestion intes-

\author{
Même avec un \\ apport suffisant de \\ fibres, des troubles \\ digestifs peuvent \\ apparaître si \\ l'apport d'amidon \\ est trop important.
}


tinale de l'amidon dépend fortement de l'âge de l'animal, puisque l'équipement enzymatique permettant l'hydrolyse de l'amidon dans l'intestin grêle n'est totalement installé qu'à pourquoi la quantité d'amidon excrétée dans les fèces est un peu plus élevée chez le lapereau de 5 semaines que chez le lapin en fin de croissance (Blas et al 1990). Cet effet est amplifié si l'amidon ingéré est plus difficilement hydrolysable. Si l'on compare les amidons d'orge et de maïs, les pertes fécales d'amidon sont respectivement de 2,2 et 5,5\% de l'amidon ingéré chez un lapereau de 4 semaines, et de 0,5 et 2,3\% chez un lapin de 10 semaines (Gidenne et Perez 1993a).

On connaît encore très mal la quantité d'amidon digérée dans l'intestin grêle, par rapport à la quantité dégradée et fermentée par la flore dans le caecum. En effet, la digestibilité iléale est actuellement mesurable in vivo seulement chez le lapin adulte, car la technique de canulation iléale est inadaptée à des lapins de petite taille, et la technique des abattages fournit des mesures incorrectes de flux iléal. Chez l'adulte, la digestibilité iléale de l'amidon peut varier entre 93 et $99 \%$ (Gidenne 1992, Merino et Carabaño 1992), alors que la digestibilité fécale de l'amidon ne varie pas et est quasi totale (99 à $100 \%)$. La digestion caecale de l'amidon ne concerne donc que des faibles quantités d'amidon et compense totalement les variations de digestion dans l'intestin grêle. Chez le lapin en croissance (poids vif inférieur à $2 \mathrm{~kg}$ ), on ne peut mesurer que la composition de l'effluent iléal après abattage. On constate que la concentration d'amidon dans l'iléon est dépendante de la teneur en amidon du régime et de l'âge de l'animal. Ainsi, lorsque le taux d'amidon dans l'aliment passe de 16 à $25 \%$, la teneur en amidon iléal passe de 6,8 à $12,9 \%$ chez un lapereau de 38 jours d'âge, et de 3,9 à

l'apport d'énergie digestible. 6 semaines d'âge (Corring et al 1972). C'est

$6,3 \%$ chez un lapin de 49 jours d'âge ; parallèlement, la mortalité augmente significativement (Blas et al 1994).

Sans changer la quantité d'amidon et de fibres ingérée, il est possible de modifier le flux iléal d'amidon en faisant varier son origine. Gidenne et Perez (1993b) ont ainsi estimé, chez le lapin adulte, que le flux iléal d'amidon d'orge était de $0,5 \mathrm{~g} / \mathrm{j}$, et celui d'amidon de maiis de $2 \mathrm{~g} / \mathrm{j}$. En outre, cette élévation du flux iléal d'amidon influence la digestion dans le caecum : la quantité de fibres dégradée (provenant principalement de luzerne) augmente avec le flux iléal d'amidon (figure 7), et le TSMt est plus long (+ 15\%).

\section{2 / Substitution de l'amidon par des fibres rapidement fermentescibles}

Les résultats antérieurs indiquent qu'un apport de fibres de type lignocellulosique (ADF) permet de réduire la fréquence des troubles digestifs du lapin en croissance, mais au détriment de l'indice de consommation du régime qui augmente. Le niveau de sécurité d'un aliment, en termes de prévention du risque d'entérite, pourrait donc se définir uniquement à partir de sa teneur en ADF. Toutefois, il faut aussi contrôler les interactions possibles avec les apports d'amidon, et préciser si la prévention des diarrhées d'origine nutritionnelle est possible à partir d'un critère unique d'apport de fibres.

Par ailleurs, le remplacement d'une fraction de l'amidon par des constituants pariétaux à digestibilité élevée dans un aliment dont la teneur en ADF est conforme aux recommandations, pourrait être une solution à l'antagonisme croissance-sécurité alimentaire. En effet, en substituant des fibres de digestibilité élevée (pectines, hémicelluloses) à de l'amidon, on peut apporter l'énergie nécessaire à une bonne croissance, tout en réduisant les risques de troubles digestifs liés à des excès d'amidon. Les fibres digestibles favoriseraient l'activité microbienne caecale (effet barrière vis-à-vis d'agents pathogènes), tandis que les fibres peu digestes (ADF) permettraient une bonne régulation du transit digestif.

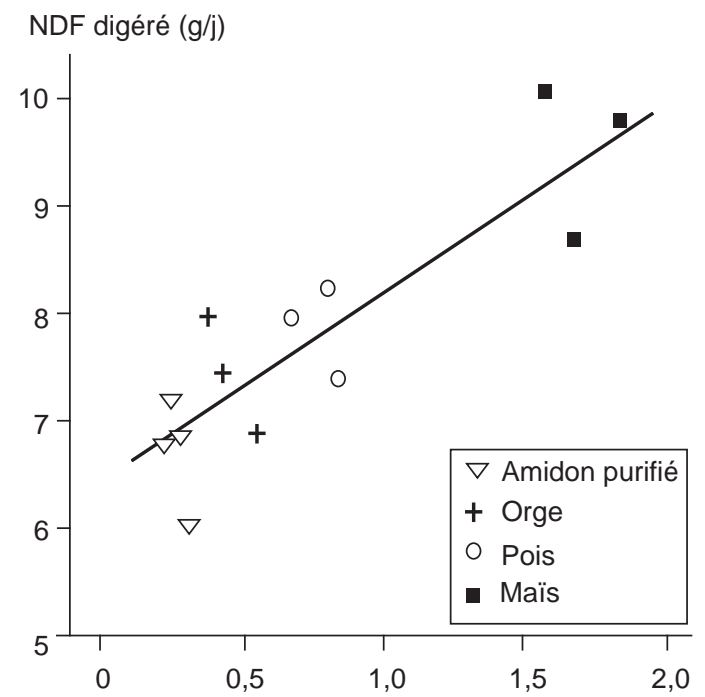

Figure 8. Substitution d'amidon par un apport $d$ 'hémicelluloses et de pectines ( $A M$ vs FD) : conséquences sur l'état sanitaire. Mesures entre 4 et 10 semaines d'âge, sur 21 lapins/régime (Jehl et Gidenne 1996).

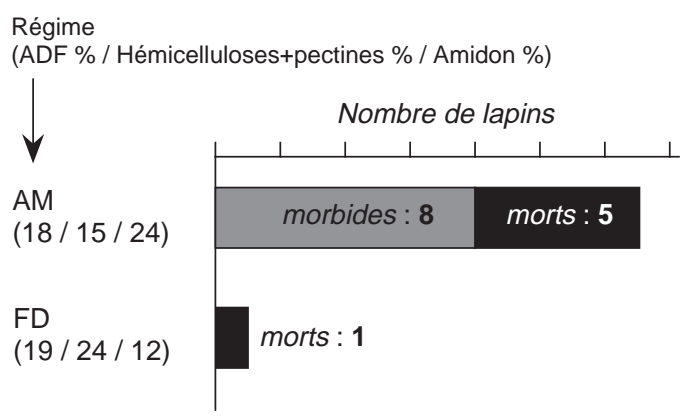


Tableau 3. Substitution d'amidon (régime AM) par un apport d'hémicelluloses et de pectines (régime $F D$ ) : conséquences sur l'indice de consommation et sur quelques paramètres digestifs. Mesures entre 4 et 10 semaines d'âge, sur 21 lapins/régime (Gidenne et Jehl 1996, Jehl et Gidenne 1996).

\begin{tabular}{|c|c|c|c|}
\hline Régime & $\begin{array}{c}\mathrm{AM} \\
(\mathrm{n}=10)\end{array}$ & $\underset{(n=10)}{F D}$ & $\begin{array}{c}\text { Signif. Stat. } \\
\text { P }\end{array}$ \\
\hline $\begin{array}{l}\text { Indice de consommation } \\
\text { Digestibilité } \mathrm{MO}(\%) \\
\mathrm{pH} \\
\text { AGV totaux }(\mathrm{mM} / \mathrm{l})\end{array}$ & $\begin{array}{l}3,01 \\
66,5 \\
6,51 \\
67,7\end{array}$ & $\begin{array}{l}3,02 \\
65,2 \\
6,23 \\
85,1\end{array}$ & $\begin{array}{c}0,87 \\
0,28 \\
0,19 \\
<0,01\end{array}$ \\
\hline
\end{tabular}

Une étude récente (Gidenne et Jehl 1996, Jehl et Gidenne 1996) a abordé ce double problème en comparant deux aliments ayant une même teneur en ADF et conforme aux recommandations actuelles (200 g ADF/kg MS), mais qui différaient par leurs teneurs en amidon et en fibres rapidement fermentescibles (hémicelluloses et pectines). Malgré une teneur en $\mathrm{ADF}$ relativement importante, une teneur élevée en amidon (régime AM) est associée à une plus grande fréquence de diarrhées (figure 8) et de profils fermentaires atypiques (inversion du ratio $\mathrm{C} 3 / \mathrm{C} 4$ ). En revanche, le régime pauvre en amidon mais contenant des fibres "digestibles "(FD) est favorable à un bon équilibre fermentaire (diminution du $\mathrm{pH}$ et élévation de la teneur en AGV), sans que l'on observe une détérioration significative de l'utilisation digestive du régime et des performances zootechniques (tableau 3). Ces résultats doivent être confirmés sur des effectifs d'animaux beaucoup plus importants. Cependant, cette étude montre que la " sécurité " d'un régime pour le lapin en croissance ne peut se définir uniquement par un critère de teneur en fibres et qu'il faut aussi prendre en compte la nature des constituants pariétaux et la teneur en amidon.

\section{Conclusion}

Les quantités d'amidon et de fibres alimentaires ingérées par le lapin influencent à la fois la digestion de l'aliment, l'activité microbienne caecale et le transit digestif (figure 9). Les effets des fibres dépendent non seulement de leur quantité mais aussi de leur nature. De même, la quantité d'amidon entrant dans le cæcum ne dépend pas seulement de la quantité ingérée mais aussi de la nature de l'amidon. Aussi, le niveau de "sécurité " d'un régime destiné à des lapins en croissance devrait être défini à la fois par une norme d'apport de fibres et d'amidon, tenant compte du stade physiologique de l'animal.

De nombreux travaux sont encore nécessaires pour fixer des recommandations à partir de résultats expérimentaux fiables, en par-

Figure 9. Incidences digestives d'une réduction du ratio fibre/amidon chez le lapin en croissance.

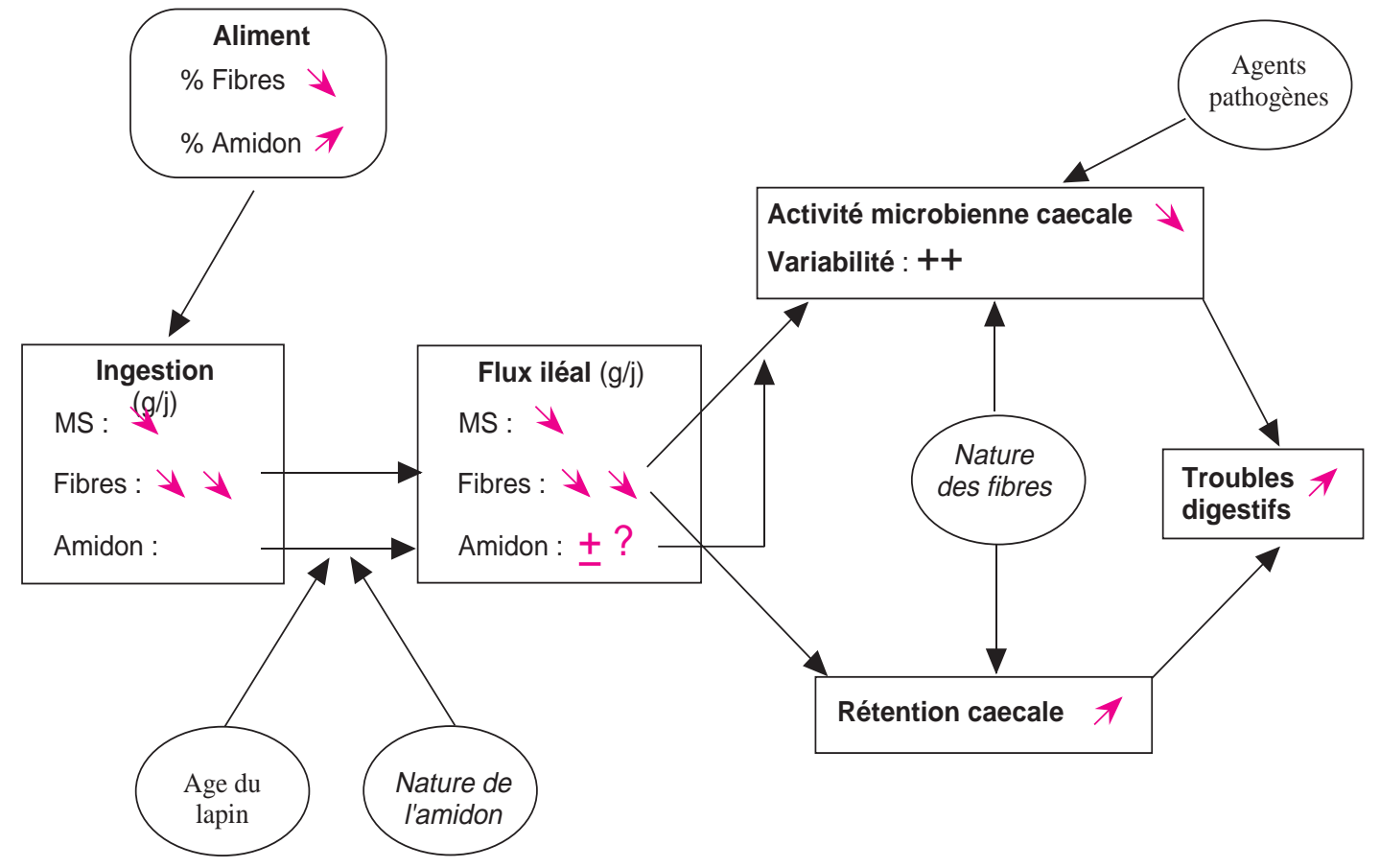


ticulier dans le cas du lapereau en période péri-sevrage, pour lequel les quelques données expérimentales actuellement disponibles sont contradictoires (Duperray 1993, Messager 1993).

Par ailleurs, une seule étude a concerné les interactions entre apports de fibres et de pro- téines (De Blas et al 1981), et seuls quelques travaux ont abordé les problèmes d'interactions entre nutrition et pathologie digestive. Il conviendrait donc de développer ces études, pour mieux comprendre les conséquences digestives de l'ingestion de fibres et d'amidon chez le lapin.

\section{Références bibliographiques}

Asp N.G., Johansson C.G., 1984. Dietary fiber analysis. Nutr. Abstr. Rev., 54, 735-752.

Aspinall O.G., 1980. Chemistry of cell-wall polysaccharides. In : J Preiss (ed), The biochemistry of plants, a comprehensive treatise, Vol 3 carbohydrates : structure and function. Academic press.

Bellier R., 1994. Contrôle nutritionnel de l'activité fermentaire caecale chez le lapin. Thèse de Doctorat Ecole Nationale Supérieure Agronomique, Institut National polytechnique de Toulouse, $117 \mathrm{p}$.

Bellier R., Gidenne T., 1995. Incidence of the source of fibre on the caecal fermentation pattern in the growing rabbit. Ann. Zootech., 44 (suppl), 187.

Bellier R., Gidenne T., 1996. Consequences of reduced fibre intake on digestion, rate of passage and caecal microbial activity in the young rabbit. Br. J. Nutr. (sous presse).

Blas E., Fandos J.C., Cervera C., Gidenne T., Perez J.M., 1990. Effet de la nature et du taux d'amidon sur l'utilisation digestive de la ration chez le lapin au cours de la croissance. 5ème Journées Rech. Cunicole. ITAVI, Paris, comm. 50.1-50.9.

Blas E., Cervera C., Fernandez-Carmona J., 1994. Effect of two diets with varied starch and fibre levels on the performances of 4-7 weeks old rabbits. World Rabbit Sci., 2, 117-121.

Candau M., Bertrand B., Fioramonti J., 1978a. Variation de la digestibilité des constituants de la ration chez le lapin. CR Séances Soc. Biol., 172, 554559.

Candau M., Delpon G., Fioramonti J., 1978b. Influence de la nature des glucides membranaires sur le développement anatomofonctionnel du tractus digestif du lapin. $2^{\text {es }}$ Journées Rech. Cunicole. INRA, Toulouse, 1.1-1.4.

Carré B., 1991. The chemical and biological bases of a calculation system developped for predicting dietary energy values : a poultry model. In : M.F Fuller (ed), In vitro digestion for pigs and poultry,67-85. $\mathrm{CAB}$ international, Wallingford, UK.

Carré B., Brillouet J.M., 1989. Determination of water-insoluble cell - walls in feeds : interlaboratory study. J. Assoc. Off. Anal. Chem., 72, 463-467.

Cheeke P.R., Patton N.M., 1980. Carbohydrate overload of the hindgut. A probable cause of enteritis. J. Appl. Rabbit Res., 3, 20-23.

Corring T., Lebas F., Courtot D., 1972. Contrôle de l'évolution de l'équipement enzymatique du pancréas exocrine du lapin de la naissance à 6 semaines. Ann. Biol. anim. Bioch. Biophys., 12, 221-231.
De Blas J.C., Carabano R., 1996. A review on the energy value of sugar beet pulp for rabbits. World Rabbit Sci., 4, 33-36.

De Blas J.C., Pérez E., Fraga M.J., Rodriguez M., Galvez J.F., 1981. Effect of diet on feed intake and growth of rabbits from weaning to slaughter at different ages and weights. J. Anim. Sci., 52, 1225-1232.

De Blas J.C., Santoma G., Carabaño, R., Fraga M.J., 1986. Fiber and starch level in fattening rabbit diets. J. Anim. Sci., 63, 1897-1904.

De Blas J.C., Villamide M.J., Carabaño R., 1989. Nutritive value of cereal by-products for rabbits. 1) Wheat straw. J. Appl. Rabbit Res., 12, 148-151.

Duperray J., 1993. Intérêt d'un aliment péri-sevrage dans l'optimisation d'un programme alimentaire. Cuniculture, 110, 79-82.

Falcao e Cunha L., 1988. Os constiutentes do parede celular no processo digestivo de colho. Thèse de docteur en agronomie. Inst.Sup.agronomia, Univ.Tecnica Lisbonne, 359 p.

Fraga M.J., Barreno C., Carabaño R., Mendez, J., De Blas J.C., 1984. Effect of amounts of fibre and protein in the diet on growth and digestive traits of rabbits. An. Inst. nac. Invest. agrar., Ganadera, 21, 91110.

Fraga M.J., Perez A.P., Carabaño R., 1991. Effect of type of fiber on the rate of passage and on the contribution of soft feces to the nutrient intake of finishing rabbits. J. Anim. Sci., 69, 1566-1574.

Franck Y., Coulmin J.P., 1978. Utilisation de la paille de blé broyée comme source de cellulose dans les aliments de lapins à l'engraissement ; comparaison de deux taux de cellulose. 2ème Journée Rech. Cunicole. INRA Toulouse, comm. $\mathrm{n}^{0} 10$.

Franck Y., Séroux M., 1980. Utilisation de la pulpe de betterave déshydratée par le lapin à l'engraissement. Proc. 2d Congress of the World Rabbit Sci. Assoc., Barcelone, WRSA ed., 2, 167-175.

Gidenne T., 1986. Evolution nycthémérale des produits de la fermentation bactérienne dans le tube digestif du lapin en croissance. Relations avec la teneur en lignines de la ration. Ann. Zootech., 35, 121-136.

Gidenne T., 1987. Effet de l'addition d'un concentré riche en fibres dans une ration à base de foin, distribuée à deux niveaux alimentaires chez la lapine adulte. 2/ Mesures de digestibilité. Reprod. Nutr. Develop., 27, 801-810.

Gidenne T., 1992. Effect of fibre level, particle size and adaptation period on digestibility and rate of 
passage as measured at the ileum and in the faeces in the adult rabbit. Br. J. Nutr., 67, 133-146.

Gidenne T., 1994. Effets d'une réduction de la teneur en fibres alimentaires sur le transit digestif du lapin. Comparaison et validation de modèles d'ajustement des cinétiques d'excrétion fécale des marqueurs. Repr. Nutr. Develop., 34, 295-306.

Gidenne T., 1995. Effect of fibre level reduction and gluco-oligosaccharide addition on the growth performance and caecal fermentation in the growing rabbit. Anim. Feed Sci. Technol., 56, 253-263.

Gidenne T., Jehl N., 1996. Replacement of starch by digestible fibre in the feed for the growing rabbit. 1) Consequences on digestibility and on rate of passage. Anim. Feed Sci. Technol., (in press).

Gidenne T., Perez J.M., 1993a. Effect of dietary starch origin on digestion in the rabbit. 1. Digestibility measurements from weaning to slaughter. Anim. Feed Sci. Technol., 42, 237-247.

Gidenne T., Perez J.M., 1993b. Effect of dietary starch origin on digestion in the rabbit. 2. Starch hydrolysis in the small intestine, cell wall degradation and rate of passage measurements. Anim. Feed Sci. Technol., 42, 249-257.

Gidenne T., Perez J.M., 1994. Apports de lignines et alimentation du lapin en croissance. I. Conséquences sur la digestion et le transit. Ann Zootech., 43, 313322 .

Gidenne T., Poncet C., Gomez L., 1987. Effet de l'addition d'un concentré riche en fibres dans une ration à base de foin, distribuée à deux niveaux alimentaires chez la lapine adulte. 1/ Temps de séjour moyen des aliments. Reprod. Nutr. Develop., 7, 733743 .

Gidenne T., Marchais C., Scalabrini F., 1990. Effet du taux et de la finesse de broyage de la luzerne chez le lapin en croissance. 2) Développement digestif, activité fermentaire cæcale et transit. $5^{\text {e Journée }}$ Rech. Cunicole. ITAVI, Paris, comm. 52.1-52.11.

Gidenne T., Scalabrani F. Marchais C., 1991. Adaptation digestive du lapin à la teneur en constituants pariétaux du régime. Ann. Zootech., 40, 73-84.

Giger-Reverdin S., 1995. Review of the main methods of cell wall estimation : interest and limits for ruminants. Anim. Feed Sci. Technol., 55, 295-334.

Heckmann F.W., 1972. Potatœ meal in complete feed of the fattening young rabbit. Arch. Gefluegelk., 36, $182-185$.

Heckmann F.W., Mehner R.A., 1970. Protein and crude fiber contents of mixed feeds for fattening young rabbits. Arch. Geflügelzucht Kleintierk., 19, $29-43$

Jehl N., Gidenne T., 1996. Replacement of starch by digestible fibre in the feed for the growing rabbit. 2) Consequences on microbial activity in the cæcum and on incidence on digestive disorders. Anim. Feed Sci. Technol., (in press).

Kœhl P.F., 1995. Gestion technico-économique nationale 1994. Cuniculture, 22, 1-5.

Laplace J.P., 1978. Le transit digestif chez les monogastriques. 3) Comportement (prise de nourriture, caecotrophie), motricité et transit digestif et pathogénie des diarrhées chez le lapin. Ann. Zootech., 27, 225-265.
Laplace J.P., Lebas F., 1977. Le transit digestif chez le lapin. 7) Influence de la finesse de broyage des constituants d'un aliment granulé. Ann. Zootech., 26, 413-420.

Lebas F., Laplace J.P., 1977. Growth and digestive transit in the rabbit. Variations determined by physical form, composition and crude fiber content of the feed. Ann. Biol. anim. Bioch. Biophys., 17, 535-53.

Lebas F., Maitre I., Arveux P., Bouillet A., Bourdillon A., Duperray J., Saint-Cast Y.,, 1989. Taux d'hémicellulose et performances de croissance du lapin de chair. Revue alimentation animale, 27, 40-43.

Lee S.C., Prosky L., Vries J.W., 1992. Determination of total, soluble, and insoluble dietary fiber in foodsenzymatic-gravimetric method, MES-TRIS buffer : collaborative study. J. Assoc. Anal. Chem., 75, 395416.

Maertens L., Mœrmans R., De Groote G., 1988. Prediction of the apparent digestible energy (ADE) content of commercial pelleted feeds for rabbits. J. Appl. Rabbit Res., 11, 60-67.

Maître I., Lebas F., Arveux P., Bourdillon A., Duperray J., Saint Cast Y., 1990. Taux de lignocellulose (ADF de Van Soest) et performances de croissance du lapin de chair. 5e Journée Rech. Cunicole. ITAVI, Paris, vol 2, 56.1-56.11.

Marounek M., Vovk S.J., Skrivanova V., 1995. Distribution of activity of hydrolytic enzymes in the digestive tract of rabbits. Br. J. Nutr., 73, 463-469.

Martinez Pascual J., Fernandez Carmona J., 1981. Citrus pulp in diets for fattening rabbits. Anim. Feed Sci. Technol., 5, 23-31.

Merino J.M., Carabaño R., 1992. Effect of type of fibre on ileal and fecal digestibility. Proc. 5th congress of the World Rabbit Science Assoc., 25-30 july, Corvallis, Oregon, USA. J. Appl. Rabbit Res., 15, 931-937.

Messager B., 1993. Un aliment de présevrage réservé aux lapereaux. Cuniculture, 110, 77-78.

Monties B. (ed), 1980. Les lignines. In : Les polymères végétaux. Gautiers-Villars, Paris.

Motta Ferreira W., 1990. Effectos de la sustitucion partial de heno de alfalfa per orujo de uva o pulpa de remolacha, sobre la utilizacion de la dieta y los rendimientos en conejos en crecimiento. Tesis Doctoral. Univ. Politec. Madrid, 251 p.

Ortiz V., De Blas J.C., 1989. Prediction of digestibility of energy of feeds for rabbits from fibre content. Invest. Agr. Prod. Sanidad Anim., 4, 197-205.

Parigi Bini R., Xiccato G., Cinetto M., 1990. Effect of dietary starch on the productivity, digestibility, body composition of growing rabbit. Zootechnica e Nutrizione Animale., 16, 271-282.

Partridge G.G., Garthwaite P.H., Findlay M., 1989. Protein and energy retention by growing rabbits offered diets with increasing proportions of fibre. J. Agric. Sci., 112, 171-178.

Peeters J.E., Charlier G.J., 1984. Le complexe entérite du lapin de chair en élevage rationnel. CuniSciences, 2, 13-26.

Perez J.M., 1990. Prévision de l'énergie digestible des aliments pour le porc : intérêt du dosage des 
parois végétales par voie enzymatique. INRA Prod Anim., 3, 171-179.

Perez J.M., Gidenne T., Lebas F., Caudron I., Arveux P., Bourdillon A., Duperray J., Messager B., 1994. Apports de lignines et alimentation du lapin en croissance. II. Conséquences sur les performances de croissance et la mortalité. Ann. Zootech., 43, 323-332.

Perez de Ayala P., Fraga M.J., Carabaño R., De Blas C., 1991. Effect of fibre source on diet digestibility and growth in fattening rabbits. J. Appl. Rabbit Res., 14, 159-165.

Selvandran R.R., 1987. Dietary fiber : chemistry, analysis and properties. In : C.O. Chixhester (ed), Advances in food research, 117-212. Academic Press.

Van Sœst P.J., Robertson J.B., Lewis B.A., 1991. Methods for dietary fiber, neutral detergent fiber, and non starch polysaccharides in relation to animal nutrition. J. Dairy Sci., 74, 3583-3597.

\begin{abstract}
Digestive effects of fibre and starch intake in the growing rabbit.

Rabbits can efficiently utilise low-fibre diets containing high contents of digestible energy. Lowering the dietary fibre level, generally associated with an increased starch level, leads to an increased incidence of digestive disturbances, which are frequently lethal for growing rabbits. Dietary fibre and starch levels must then be controlled to allow for both optimal growth rates and feed security. An increase in lignocellulose (ADF, slightly degraded) reduces the feed digestibility and stimulates the digesta rate of passage, without greatly affecting the caecal fermentations. An increase in more easily digestible fibre (hemicellulose + pectins) slightly affects the feed digestibility, but dœs not slow the rate of passage ; on the other hand it promotes caecal microbial activity (CMA). An ADF increase seems efficient in reducing the incidence of digestive troubles and mortality during the fattening period.
\end{abstract}

Abstract
The CMA can also be affected by the quantity of starch entering the caecum, itself dependent on the nature of the starch and the age of the animal. Increasing the dietary starch level could promote digestive troubles, even when ADF level is in agreement with current recommendations.

Thus, the "security level" of a feed can not only be defined by a minimum level of ADF, but must also take into account a maximum level of starch. The replacement of starch by easily digestible cellwall constituents, in a diet having a minimum level of lignocellulose, could be a solution to the antagonism between efficiency and feed security in the growing rabbit.

GIDENNE T., 1996. Conséquences digestives de l'ingestion de fibres et d'amidon chez le lapin en croissance : vers une meilleure définition des besoins. INRA Prod. Anim., 9 (4), 243-254. 